\title{
Anti-allergic effect of Artemisia extract in rats
}

\author{
YAN DENG, ZIJUN LIU and YIWEI GENG
}

School of Traditional Chinese Medicine, Southern Medical University, Guangzhou, Guangdong 510515, P.R. China

Received January 22, 2015; Accepted February 25, 2016

DOI: $10.3892 /$ etm.2016.3361

\begin{abstract}
Artemisiaapiacea (also known as Artemisia annua L) is a herb commonly used in traditional Chinese medicine. In the early $1970 \mathrm{~s}$, artemisinin was isolated and identified as the active antimalarial ingredient, and thereafter, A. apiacea and artemisinin have been studied extensively, such as anti-inflammation and antipyresis, antibacteria, antiparasitic and immunosuppression effects of A. apiacea extract. The present study investigated the extracts anti-allergic effect obtained from the dried flowering tips of A. apiacea in rats. A systemic anaphylactic reaction model was induced in rats using compound 48/80. Artemisia extract was administered $1 \mathrm{~h}$ prior to the injection of compound 48/80. Artemisia was extracted from dried flowering tips of $A$. deserti using $80 \%$ ethanol. Subsequently, the systemic anaphylactic shock, histamine release, scratching behavior and vascular permeability induced by compound 48/80 were evaluated. The administration of Artemisia extract at 200 and $400 \mathrm{mg} / \mathrm{kg}$ doses suppressed the systemic anaphylactic shock induced by compound 48/80 in a dose-dependent manner. Overall, the Artemisia extract was able to effectively decrease systemic anaphylactic shock, histamine release, scratching behavior and vascular permeability induced by compound 48/80 in a dose-dependent manner.
\end{abstract}

\section{Introduction}

Hypersensitivity reaction is a type of B-type adverse reaction, which is an abnormal reaction unrelated to dose and conventional pharmacological effects and unpredictable in preclinical experiments, with low incidence and high mortality (1). Hypersensitivity reaction is referred to as anaphylaxis (also known as Type I hypersensitivity) and is a strong reaction produced by the body when the immune system is stimulated by a certain allergen repeatedly. Type I

Correspondence to: Ms. Yan Deng or Dr Zijun Liu, School of Traditional Chinese Medicine, Southern Medical University, 1023 Shatai Road, Guangzhou, Guangdong 510515, P.R. China E-mail: ydengjs2014@163.com

E-mail: 396337999@qq.com

Key words: artemisia extract, systemic anaphylactic reaction, allergic models hypersensitivity is usually caused by an immunogenic macromolecule substance or hapten molecules capable of being combined with a macromolecular carrier (2). Allergens may stimulate lymphocytes to generate antibody $\operatorname{IgE}$, which is combined with high-affinity IgE receptor (FceRI) of mast cells or basophils via the circulatory system. Once the allergen enters the body, its combination with IgE leads to FceRI coupling, thus triggering the degranulation of mast cells or basophils, and the release of allergic mediators causes local or systemic reactions (3).

The majority of drug-induced allergic reactions are Type I hypersensitivity reactions ranging from the local to the body; local reactions include allergic dermatitis, and systemic allergic reactions may lead to anaphylactic shock and patient fatality (4). Artemisinin is an antimalarial active chemical component isolated from Artemisia extract.Artemisia apiacea (also known as Artemisia annua L) contains $>140$ types of chemical substance, of which $>30$ have anti-malarial activity. A recently identified chemical substance in A. apiacea extract, isolated by the China Traditional Chinese Medicine Academy (http://www.catcm.ac.cn/), has shown a good antimalarial effect that is equal to artemisinin (5-7). A. apiacea is a herb commonly used in traditional Chinese medicine, which exhibits anti-inflammatory and antipyresis, antibacterial, antiparasitic and immunosuppressive effects (8). Therefore, the aim of the present study was to evaluate the effect of Artemisia extract on the allergic response induced by compound $48 / 80$ in rats.

\section{Materials and methods}

Animals and grouping. A total of 60 male Wistar rats (weight, 230-280 g; age, 7 weeks) were obtained from the Animal Experimental Center of Southern Medical University (Guangzhou, China) and were maintained at a constant temperature $\left(24 \pm 2^{\circ} \mathrm{C}\right)$ with a relative humidity of $55 \pm 15 \%$ in a 12-h light/dark cycle. All rats were administered standard laboratory rodent feed and water ad libitum. A total of 50 rats were randomly assigned into 5 groups: A spontaneous group $(\mathrm{n}=10)$, a control $(\mathrm{n}=10), 100 \mathrm{mg} / \mathrm{kg}$ of Artemisia extract-treated group $(\mathrm{n}=10), 200 \mathrm{mg} / \mathrm{kg}$ of Artemisia extract-treated group $(\mathrm{n}=10), 400 \mathrm{mg} / \mathrm{kg}$ of Artemisia extract-treated group $(\mathrm{n}=10)$. This study was in accordance with the Southern Medical University Guidelines and Regulations on the Use and Care of Lab Animals. Ethical approval was obtained for this study from the Southern Medical University. 
Preparation of the extract. The dried flowering tips of A. apiacea were obtained from Kunming Institute of Medicine (Kunming, China) and were ground into fine powder using an electric blender (Thermo Fisher Scientific Inc., Waltham, MA, USA). Next, $20 \mathrm{~g}$ powder was ground and refluxed with $80 \%$ ethanol $(600 \mathrm{ml})$ using a Soxhlet extractor (Thermo Fisher Scientific Inc.) for $8 \mathrm{~h}$. The extraction process was repeated twice. The extract was separated by passing the mixture through filter paper. and the resulting Artemisia extract was freshly dissolved in a 5\% gum arabic (Sinopharm Chemical Reagent Co., Ltd., Shanghai, China) solution prior to use.

Compound 48/80-induced systemic anaphylactic reaction. All rats received anintraperitoneal(i.p.)injection of compound 48/80 (8 mg/kg; Sigma-Aldrich, St. Louis, MO, USA ) to induce a systemic anaphylactic reaction. The systemic anaphylactic reaction model was conducted according to the method previously described by Shin et al (9). Artemisia extract was administered $1 \mathrm{~h}$ prior to the injection of compound 48/80. Following the induction of anaphylactic shock, mortality rate was recorded over a 1-h period.

Compound 48/80-induced histamine release from isolated rat peritoneal mast cells. The rats were cervically dislocated under anesthesia with $40 \mathrm{mg} / \mathrm{kg}$ pentobarbital sodium). Peritoneal mast cells were harvested from male Wistar strain rats using decollation under $350 \mathrm{mg} / \mathrm{kg}$ chloral hydrate. A total of $5 \mathrm{ml}$ of medium was injected into enterocoelia of every mouse and the abdomen was gently massaged for 5-10 min. The cells were purified using Percoll density centrifugation (Thermo Fisher Scientific, Inc.) at $8,000 \mathrm{xg}$ at $4^{\circ} \mathrm{C}$ for $10 \mathrm{~min}$, as described previously (10). All cells $\left(3 \times 10^{4}\right.$ cells/tube) were incubated in physiological buffer solution (Beyotime Institute of Biotechnology, Haimen, China) for $20 \mathrm{~min}$ at $37^{\circ} \mathrm{C}$. Artemisia extract, $0.1 \mathrm{ml}$ phosphate-buffered saline (Wuhan Procell Science and Technology Co., Ltd., Wuhan, China) and $0.5 \mathrm{mg} / \mathrm{ml}$ compound $48 / 80$ were blended altogether. The miscible liquids were incubated for $20 \mathrm{~min}$ on ice. The histamine content was measured by means of a fluorometric assay (ELISTA kit; E-EL-0032c, Elabscience, Wuhan, China) by an ELx800 microplate reader (Biotek Instruments, Inc., Winooski, VT, USA). Briefly, the miscible liquids were incubated for $20 \mathrm{~min}$ on ice and $80 \mu \mathrm{l}$ of affinity chain enzyme-HRP at $37^{\circ} \mathrm{C}$ was added for $1 \mathrm{~h}$. Next, every well was washed with scrubbing solution. The histamine content was measured by means of a fluorometric assay (Synergy 2 Microplate Reader, Bio-Tek, USA) at $450 \mathrm{~nm}$.

Scratching behavior. Scratching behavior was surveyed using a previously described method (11). The Artemisia extract was administered orally and scratching behavior was observed for $1 \mathrm{~h}$. Next, $10 \mu \mathrm{g} / 0.02 \mathrm{ml}$ compound 48/80, $100 \mathrm{nmol} / 0.02 \mathrm{ml}$ histamine and $100 \mathrm{nmol} / 0.02 \mathrm{ml}$ serotonin (both from Sigma-Aldrich) were injected intradermally into the rostral part of the back of the rats. Scratching behavior of rat was observed for a further $1 \mathrm{~h}$, and evaluated using a HF-200 camera (Canon Legria, Canon Inc., Tokyo, Japan).

Vascular permeability of the skin. Artemisia extract was administered orally and $1 \mathrm{~h}$ passed before subsequent
Table I. Effect of Artemisia extract on the systemic anaphylactic shock induced by compound $48 / 80$.

\begin{tabular}{lccc}
\hline & \multicolumn{3}{c}{ Mortality rate (\%) } \\
\cline { 2 - 4 } Group & 10 min & 20 min & 60 min \\
\hline Control & 10 & 60 & 100 \\
Artemisia extract $(\mathrm{mg} / \mathrm{kg})$ & & & \\
100 & 0 & 30 & 70 \\
200 & 0 & 20 & 50 \\
400 & 0 & 10 & 40 \\
\hline
\end{tabular}

Mortality within $1 \mathrm{~h}$ after compound 48/80 injection is presented as the number of dead rats/total number rats $(n=10)$.

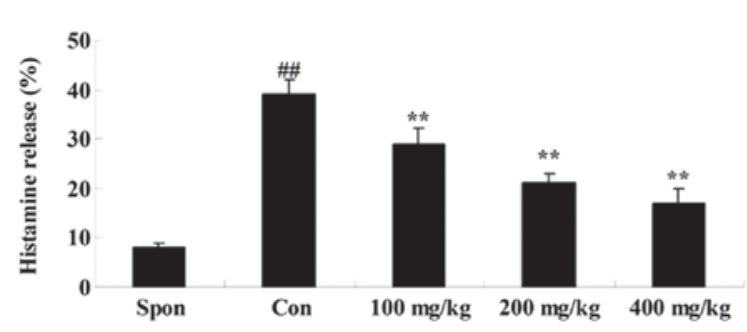

Figure 1. Effect of artemisia extract on the histamine release from rat peritoneal mast cells induced by compound $48 / 80$. ${ }^{\# \#} \mathrm{P}<0.01$ vs. Spon group, ${ }^{* *} \mathrm{P}<0.01$ vs. Con group. Spon, spontaneous-group; Con, control-group; $100 \mathrm{mg} / \mathrm{kg}$, Artemisia extract (100 mg/kg)-treated group; $200 \mathrm{mg} / \mathrm{kg}$, Artemisia extract (200 mg/kg)-treated group; $400 \mathrm{mg} / \mathrm{kg}$, Artemisia extract (400 mg/kg)-treated group. Statistical analysis was done with the Student's t-test and one-way analysis of variance followed by Dunnett's test.

experiments. Next, $0.5 \mathrm{mg} / 0.02 \mathrm{ml}$ compound 48/80 (i.p.), $10 \mathrm{nmol} / 0.02 \mathrm{ml}$ histamine (i.p.) or $10 \mathrm{nmol} / 0.02 \mathrm{ml}$ serotonin (i.p.) was administered into the rostral part of the back. In addition, 2\% Evans blue solution (Beyotime Institute of Biotechnology) was intravenously injected into each animal. After a pentobarbital sodium injection (1.5\%; Sigma-Aldrich) the rats were sacrificed using cervical dislocation and after $30 \mathrm{~min}$, then the 'bluing' reaction diameter was measured at the injection site.

Statistical analysis. Data are presented as the mean \pm standard error of the mean. Statistical analyses were performed using SPSS 17.0 software (SPSS, Inc., Chicago, IL, USA). Statistical significance was tested using one-way analysis of variance followed by Dunnett's test. $\mathrm{P}<0.05$ was considered to indicate a statistically significant difference.

\section{Results}

Effect of Artemisia extract on the systemic anaphylactic shock induced by compound 48/80. To determine the effect of artemisia extract on the systemic anaphylactic shock induced by compound $48 / 80$, the mortality rate within $1 \mathrm{~h}$ after compound 48/80 injection was recorded. The administration of $8 \mathrm{mg} / \mathrm{kg}$ compound 48/80 (i.p.) increased the mortality rate in the allergic model rats (Table I). However, the Artemisia extract pretreatment reduced the mortality rate of allergic 


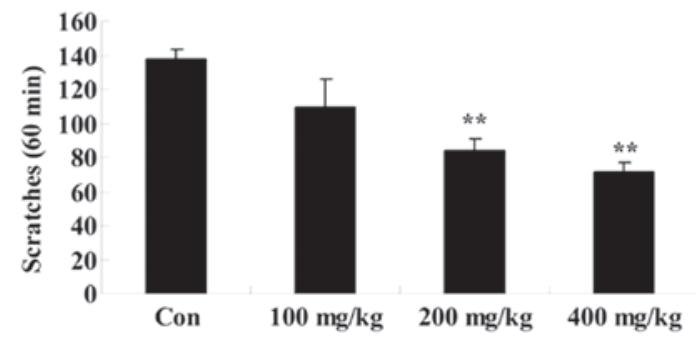

Figure 2. Effect of Artemisia extract on the scratching behavior. ${ }^{* *} \mathrm{P}<0.01$ vs. Con group. Con, control-group; $100 \mathrm{mg} / \mathrm{kg}$, Artemisia extract $(100 \mathrm{mg} / \mathrm{kg}$ )-treated group; $200 \mathrm{mg} / \mathrm{kg}$, Artemisia extract (200 mg/kg)-treated group; $400 \mathrm{mg} / \mathrm{kg}$, Artemisia extract $(400 \mathrm{mg} / \mathrm{kg})$-treated group. Statistical analysis was done with the Student's t-test and one-way analysis of variance followed by Dunnett's test.

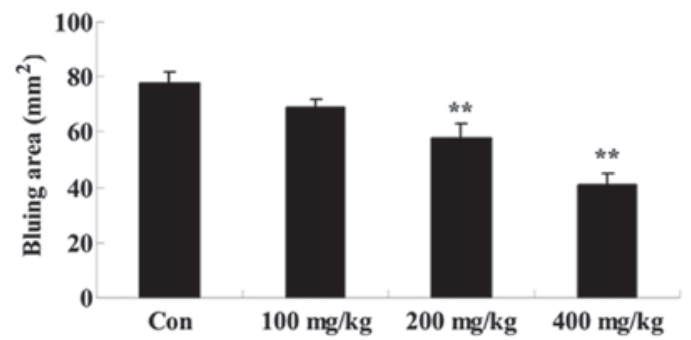

Figure 3. Effect of Artemisia extract on the vascular permeability. ${ }^{* *} \mathrm{P}<0.01$ vs. Con group.Con, control-group; $100 \mathrm{mg} / \mathrm{kg}$,Artemisia extract $(100 \mathrm{mg} / \mathrm{kg})$-treated group; $200 \mathrm{mg} / \mathrm{kg}$, Artemisia extract (200 mg/kg)-treated group; $400 \mathrm{mg} / \mathrm{kg}$, Artemisia extract ( $400 \mathrm{mg} / \mathrm{kg}$ )-treated group. Statistical analysis was done with the Student's t-test and one-way analysis of variance followed by Dunnett's test.
A

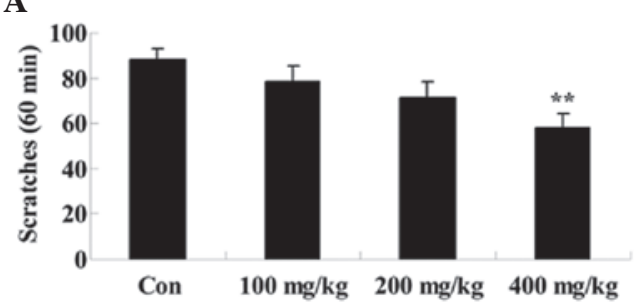

B

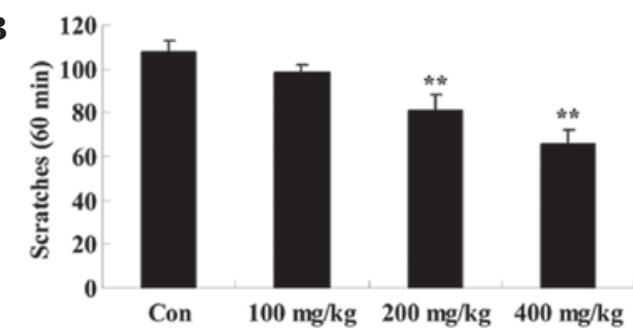

Figure 4. Effects of Artemisia extract on the scratching behavior induced by histamine and serotonin. Effects of artemisia extract on the scratching behavior induced by (A) histamine and (B) serotonin. ${ }^{* *} \mathrm{P}<0.01$ vs. Con group. Con, control-group; $100 \mathrm{mg} / \mathrm{kg}$, Artemisia extract (100 mg/kg)-treated group; $200 \mathrm{mg} / \mathrm{kg}$, Artemisia extract (200 mg/kg)-treated group; $400 \mathrm{mg} / \mathrm{kg}$, Artemisia extract ( $400 \mathrm{mg} / \mathrm{kg}$ )-treated group. Statistical analysis was done with the Student's t-test and one-way analysis of variance followed by Dunnett's test.
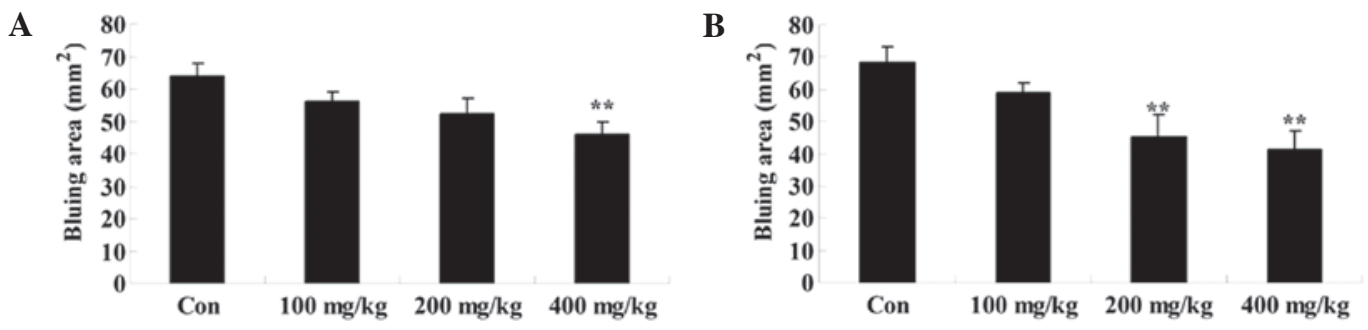

Figure 5. Effect of Artemisia extract on the vascular permeability induced by histamine and serotonin. Effects of Artemisia extract on the vascular permeability induced by (A) histamine and (B) serotonin. ${ }^{* * *} \mathrm{P}<0.01$ vs. Con group. Con, control-group; $100 \mathrm{mg} / \mathrm{kg}$, Artemisia extract (100 mg/kg)-treated group; $200 \mathrm{mg} / \mathrm{kg}$, Artemisia extract ( $200 \mathrm{mg} / \mathrm{kg}$ )-treated group; $400 \mathrm{mg} / \mathrm{kg}$, Artemisia extract (400 mg/kg)-treated group. Statistical analysis was done with the Student's t-test and one-way analysis of variance followed by Dunnett's test.

model rats in a concentration- and time-dependent manner (Table I).

Effect of Artemisia extract on the histamine released from rat peritoneal mast cells induced by compound 48/80. A fluorometric assay was performed to investigate the effect of Artemisia extract on histamine release in the allergic model rats. Treatment with $0.5 \mathrm{mg} / \mathrm{ml}$ compound $48 / 80$ significantly increased the histamine release in the allergic model rats (Fig. 1). Artemisia extract pretreatment (100, 200 and $400 \mathrm{mg} / \mathrm{kg}$ ) significantly reduced the histamine release in the allergic model rats, compared with the untreated control (Fig. 1).

Effect of Artemisia extract on the scratching behavior induced by compound 48/80. To elucidate the effect of Artemisia extract on the scratching behavior of allergic model rats, the scratching behavior was induced by treatment with compound 48/80. Furthermore, the Artemisia extract pretreatment (200 and $400 \mathrm{mg} / \mathrm{kg}$ ) significantly reduced the scratching behavior of the allergic model rats (Fig. 2).

Effect of Artemisia extract on the vascular permeability induced by compound 48/80. To assess the effect of Artemisia extract on the vascular permeability of allergic model rats, the vascular permeability was evaluated following $0.5 \mathrm{mg} / 0.02 \mathrm{ml}$ compound 48/80 injection. Compound 48/80 effectually augmented the vascular permeability of allergic model rats (Fig. 3). The Artemisia extract pretreatment (200 and $400 \mathrm{mg} / \mathrm{kg}$ ) significantly reduced the vascular permeability of allergic model rats (Fig. 3).

Effects of Artemisia extract on the scratching behavior induced by histamine and serotonin. To examine the effect of Artemisia extract on the scratching behavior of allergic model rats, scratching behavior was induced with $100 \mathrm{nmol} / 0.02 \mathrm{ml}$ 
histamine or serotonin injection. Histamine or serotonin significanlty increased the scratching behavior of allergic model rats (Fig. 4A and B). The Artemisia extract pretreatment (400 mg/kg) significantly reduced the scratching behavior induced by histamine (Fig. 4A). Furthermore, the Artemisia extract pretreatment (200 and $400 \mathrm{mg} / \mathrm{kg}$ ) significantly reduced the scratching behavior induced by serotonin (Fig. 4B).

Effect of artemisia extract on the vascular permeability induced by histamine and serotonin. To analyze the effect of Artemisia extract on the vascular permeability of allergic model rats, the vascular permeability was investigated after $10 \mathrm{nmol} / 0.02 \mathrm{ml}$ histamine or serotonin injection. Histamine or serotonin markedly increased the vascular permeability of allergic model rats (Fig. 5A and B). The Artemisia extract pretreatment $(400 \mathrm{mg} / \mathrm{kg})$ significantly reduced the vascular permeability induced by histamine (Fig. 5A). Furthermore, the Artemisia extract pretreatment (200 and $400 \mathrm{mg} / \mathrm{kg}$ ) significantly reduced the vascular permeability induced by serotonin (Fig. 5B).

\section{Discussion}

Hypersensitivity reaction is a type of B-type adverse reaction that is an abnormal reaction unrelated to dose and conventional pharmacological effects, and is unpredictable in preclinical experiments, with low incidence and high mortality (12). Drug-induced allergic reactions may be serious, as anaphylactic shock may lead to patient fatality (13). Establishing a sensitive and reliable animal model for the study of allergic anaphylaxis is necessary; as a basis for screening and discovering drug allergens in addition to the investigation of drug-induced allergic reaction mechanisms, that are relevant to the prevention and treatment of clinical allergic reactions to drugs (14). However, during the preclinical safety evaluation of drugs, allergic reaction is among the most difficult factors to predict due to the lack of appropriate models for predicting clinical allergic reactions (15). The establishment of appropriate animal models for allergic reaction prediction, and their successful application to predicting drug allergies are required $(16,17)$. The present results demonstrated that Artemisia extract pretreatment is capable of reducing the systemic anaphylactic shock, histamine release, scratching behavior and vascular permeability induced by compound 48/80. Previously, Kim et al suggested that Artemisia asiatica extract improved airway inflammation of allergic asthma in mice (18).

The safety of various traditional Chinese medicines, particularly with regard to clinical allergic reaction, has led to increased their preclinical re-evaluation in China to guarantee drug safety of patients (19). The traditional Chinese medicine A. apiacea is also known as Artemisia annua L (20). Its extract is anti-allergy medicine, which has been proposed as a treatment of disease due to its speculated anti-illness effects, limited side effects and low cost (21). Recent studies show that the extract is a physiologically active traditional medicine, with anti-allergic, anti-oxidative and anti-inflammatory effects (22-24). In the present study, the anti-allergic effect of Artemisia extract markedly suppressed the scratching behavior and vascular permeability induced by histamine and serotonin.
In conclusion, the present findings suggest that the extract obtained from the dried flowering tips of A. apiacea exerts an anti-allergic effect on the allergic reaction induced by compound 48/80 in rats. This study provides an experimental basis for the investigation of Artemisia extract in the treatment of allergic diseases.

\section{Acknowledgements}

This study was supported by The National Natural Science Fund (grant no. 81202704).

\section{References}

1. Kiss B, Szántó M, Szklenár M, Brunyánszki A, Marosvölgyi T, Sárosi E, Remenyik É, Gergely P, Virág L, Decsi T, et al: Poly (ADP) ribose polymerase-1 ablation alters eicosanoid and docosanoid signaling and metabolism in a murine model of contact hypersensitivity. Mol Med Rep 11: 2861-2867, 2015.

2. Roth-Walter F, Gomez-Casado C, Pacios LF, Mothes-Luksch N, Roth GA, Singer J, Diaz-Perales A and Jensen-Jarolim E: Bet v 1 from birch pollen is a lipocalin-like protein acting as allergen only when devoid of iron by promoting $\mathrm{Th} 2$ lymphocytes. J Biol Chem 289: 17416-17421, 2014.

3. Mitoshi M, Kuriyama I, Nakayama H, Miyazato H, Sugimoto K, Kobayashi Y, Jippo T, Kuramochi K, Yoshida H and Mizushina Y: Suppression of allergic and inflammatory responses by essential oils derived from herbal plants and citrus fruits. Int J Mol Med 33: 1643-1651, 2014.

4. Kim JH, Yi JS, Gong CH and Jang YJ: Development of Aspergillus protease with ovalbumin-induced allergic chronic rhinosinusitis model in the mouse. Am J Rhinol Allergy 28: 465-470, 2014.

5. Brandys J, Grimsøen A, Nilsen BM, Paulsen BS, Park HS and Hong CS: Cross-reactivity between pollen extracts from six artemisia species. Planta Med 59: 221-228, 1993.

6. Olsen OT, Frølund L, Heinig J, Jacobsen L and Svendsen UG: A double-blind, randomized study investigating the efficacy and specificity of immunotherapy with Artemisia vulgaris or Phleum pratense/Betula verrucosa. Allergol Immunopathol (Madr) 23: 73-78, 1995.

7. Subiza J, Subiza JL, Alonso M, Hinojosa M, Garcia R, Jerez M and Subiza E: Allergic conjunctivitis to chamomile tea. Ann Allergy 65: 127-132, 1990.

8. Ryu JC, Park SM, Hwangbo M, Byun SH, Ku SK, Kim YW, Kim SC, Jee SY and Cho IJ: Methanol extract of Artemisia apiacea hance attenuates the expression of inflammatory mediators via NF- $\kappa \mathrm{B}$ inactivation. Evid Based Complement Alternat Med: Feb 22, 2013 (Epub ahead of print).

9. Shin TY, Park JH and Kim HM: Effect of Cryptotympana atrata extract on compound 48/80-induced anaphylactic reactions. J Ethnopharmacol 66: 319-325, 1999.

10. Noori A, Amjad L and Yazdani F: The effects of Artemisia deserti ethanolic extract on pathology and function of rat kidney. Avicenna J Phytomed 4: 371-376, 2014.

11. Kuraishi Y, Nagasawa T, Hayashi K and Satoh M: Scratching behavior induced by pruritogenic but not algesiogenic agents in mice. Eur J Pharmacol 275: 229-233, 1995.

12. Du Q, Gu X, Cai J, Huang M and Su M: Chrysin attenuates allergic airway inflammation by modulating the transcription factors T-bet and GATA-3 in mice. Mol Med Rep 6: 100-104, 2012.

13. Cheng LJ, Liu B, Ning B, Ming H, Wang C and Wan LX: High-intensity focused ultrasound for the treatment of allergic rhinitis using nasal endoscopy. Exp Ther Med 5: 320-322, 2013.

14. Hui Y, Li L, Qian J, Guo Y, Zhang X and Zhang X: Efficacy analysis of three-year subcutaneous SQ-standardized specific immunotherapy in house dust mite-allergic children with asthma. Exp Ther Med 7: 630-634, 2014.

15. Hollander SM, Joo SS and Wedner HJ: Factors that predict the success of cyclosporine treatment for chronic urticaria. Ann Allergy Asthma Immunol 107: 523-528, 2011.

16. Kim HH, Choi PH, Yoo JS, Jeon H, Chae BS, Park JS, Kim SH and Shin TY: Ripe fruit of Rubus coreanus inhibits mast cell-mediated allergic inflammation. Int J Mol Med 29: 303-310, 2012. 
17. Yong J, Chen GQ, Huang B and Wu S: Correlation between the ratio of T-bet/GATA-3 and the levels of IL- 4 and IFN- $\gamma$ in patients with allergic asthma. Mol Med Rep 4: 663-666, 2011

18. Kim JY, Kim DY, Lee YS, Lee BK, Lee KH and Ro JY: DA-9601, Artemisia asiatica herbal extract, ameliorates airway inflammation of allergic asthma in mice. Mol Cells 22: 104-112, 2006.

19. Guo H and Liu MP: Mechanism of traditional Chinese medicine in the treatment of allergic rhinitis. Chin Med J (Engl) 126: 756-760, 2013.

20. Zhang F, Fu X, Lv Z, Shen Q, Yan T, Jiang W, Wang G, Sun X and Tang K: Type $2 \mathrm{C}$ phosphatase 1 of Artemisia annua L. Is a negative regulator of ABA signaling. Biomed Res Int 2014: 521794, 2014.

21. Yin Y, Gong FY, Wu XX, Sun Y, Li YH, Chen T and Xu Q Anti-inflammatory and immunosuppressive effect of flavones isolated from Artemisia vestita. J Ethnopharmacol 120: 1-6, 2008
22. Rasool R, Ganai BA, Akbar S and Kamili AN: Free radical scavenging potential of in vitro raised and greenhouse acclimatized plants of Artemisia amygdalina. Chin J Nat Med 11: 377-384, 2013.

23. Park JM, Hahm KB, Kwon SO and Kim EH: The Anti-inflammatory effects of acidic polysaccharide from Artemisia capillaris on Helicobacter pylori infection. J Cancer Prev 18: 161-168, 2013.

24. Puc M and Wolski T: Forecasting of the selected features of Poaceae (R. Br.) Barnh., Artemisia L. and Ambrosia L. pollen season in Szczecin, north-western Poland, using Gumbel's distribution. Ann Agric Environ Med 20: 36-47, 2013. 